\title{
GRP78 Secreted by Colon Cancer Cells Facilitates Cell Proliferation via PI3K/Akt Signaling
}

\author{
Rong Fu' ${ }^{1 \&}$, Peng Yang ${ }^{1 \&}$, Hai-Li Wu1 ${ }^{1}$, Zong-Wei Li ${ }^{1}$, Zhuo-Yu Li1 ${ }^{12 *}$
}

\begin{abstract}
Glucose regulated protein 78 (GRP78) is usually recognized as a chaperone in the endoplasmic reticulum. However, increasing evidence indicates that GRP78 can be translocated to the cell surface, acting as a signaling receptor for a variety of ligands. Since little is known about the secretion of GRP78 and its role in the progression of colon cancer we here focused on GRP78 from colon cancer cells, and purified GRP78 protein mimicking the secreted GRP78 was able to utilize cell surface GRP78 as its receptor, activating downstream PI3K/Akt and Wnt/ק-catenin signaling and promote colon cancer cell proliferation. Our study revealed a new mode of action of autocrine GRP78 in cancer progression: secreted GRP78 binds to cell surface GRP78 as its receptor and activates intracellular proliferation signaling.
\end{abstract}

Keywords: autocrine - colon cancer - glucose regulated protein 78 - proliferation

Asian Pac J Cancer Prev, 15 (17), 7245-7249

\section{Introduction}

Glucose regulated protein 78 (GRP78) is traditionally regarded as a major molecular chaperone in the endoplasmic reticulum (ER), which facilitates protein folding and assembly, protein quality control, $\mathrm{Ca}^{2+}$ binding and regulates ER stress signaling. GRP78 is usually overexpressed in tumor cells, and implicated in tumor cell survival, angiogenesis, metastasis and therapy resistance (Lee, 2007; Vlashi et al., 2011; Li and Li, 2012). GRP78 is also involved in transcriptional activation of proinflammatory cytokine genes and is a valid therapeutic target for prevention of liver cancer (Wang et al., 2014).

Emerging evidences indicate that GRP78 can be translocated to the cell surface and acts as a receptor for a variety of ligands, exhibiting a wide range of biological effects. Ligation of surface GRP78 by activating $\alpha 2-$ macroglobulin $\left(\alpha 2-\mathrm{M}^{*}\right)$ activates MAPK and Aktdependent signaling and promotes cell proliferation of prostate cancer cells (Misra et al., 2006). Cripto protein can form a complex with GRP78 at the cell surface, inhibiting transforming growth factor signaling and enhancing cell growth (Shani et al., 2008; Kelber et al., 2009). In addition, cell surface GRP78 promotes colorectal cancer cell migration and invasion, independent of its signaling receptor function (Li et al., 2013).

Besides its location in the ER and cell membrane, GRP78 has been found present in cytoplasm, mitochondria, nucleus and cellular secretions (Vlashi et al., 2011). Some solid tumor cells are able to secrete high amounts of GRP78, which blocks the antiangiogenic activity of bortezomib through activation of extracellular signalrelated kinase and inhibition of p53 in endothelial cells (Kern et al., 2009). However, other biological roles of secreted GRP78 in tumor microenvironment and its action mode have not been identified.

In the present study, we demonstrate that GRP78 can be secreted from colon cancer cells. Recombinant GRP78 mimicking secreted GRP78 promoted cancer cell proliferation. Interestingly, purified GRP78 could utilize cell surface GRP78 as its receptor, and activate PI3K/ Akt signaling. These findings provide novel insights into understanding biological functions of secreted GRP78.

\section{Materials and Methods}

\section{Materials}

RPMI-1640 medium and fetal bovine serum (FBS) were from GIBCO (Grand Island, NY). MTT and DAPI were obtained from Sigma (St. Louis, MO). GST affinity resin (glutathione Sepharose 4 Fast Flow) was purchased from GE Healthcare (Uppsala, Sweden). Antibodies for cyclin D1, cyclin E, $\beta$-actin and GAPDH were from Bioworld Technology (Minneapolis, MN). Antibodies for $p$-GSK-3 $\beta, p$-Akt and Akt were obtained from Cell Signaling Technology (Danvers, MA). Antibodies for GRP78, $\beta$-catenin and c-Myc were from Abcam (Cambridge, UK). HRP-conjugated secondary antibodies were obtained from Invitrogen (Carlsbad, CA).

Protein purification and non-denaturing PAGE assay

Cellular RNA was extracted from HT-29 cells using

${ }^{1}$ Institute of Biotechnology, Key Laboratory of Chemical Biology and Molecular Engineering of National Ministry of Education, Shaxi University, Taiyuan, ${ }^{2}$ College of Lif Science, Zhejiang Chinese Medical University, Hangzhou, China ${ }^{\circledR}$ Equal contributors *For correspondence:lzy@sxu.edu.cn 
A

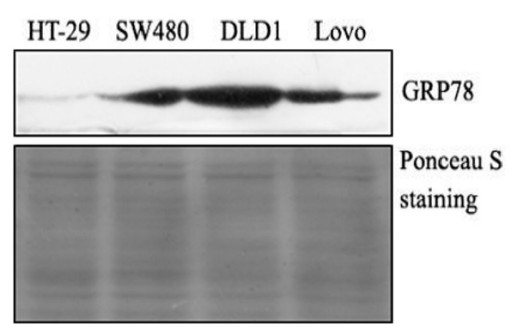

D

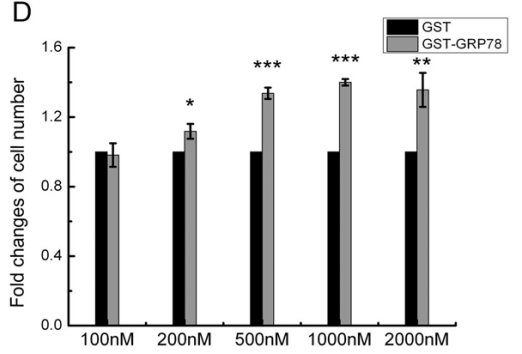

B
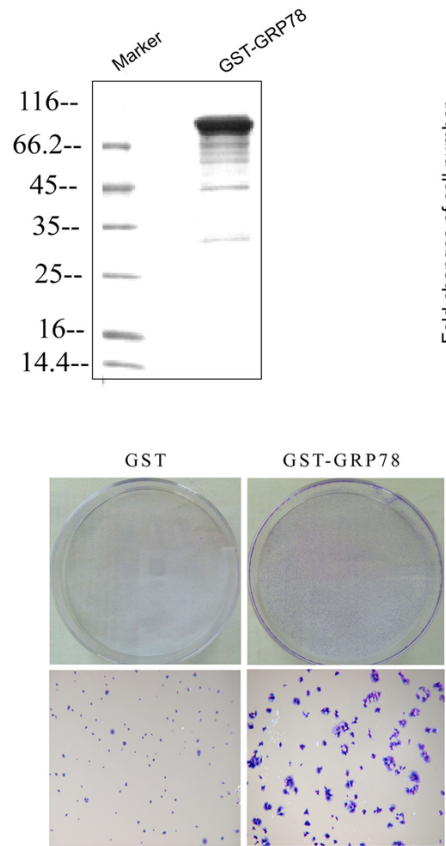
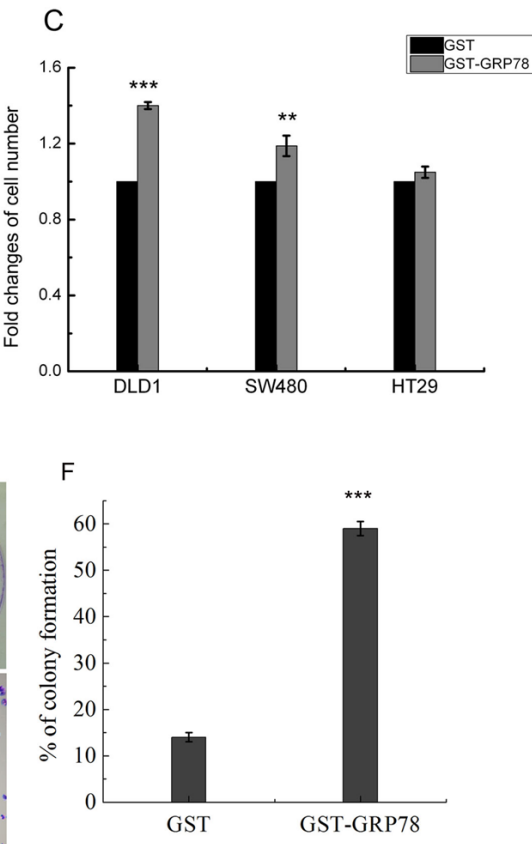

Figure 1. Secreted GRP78 Promotes Cell Proliferation. (A) Western blotting detection of GRP78 in secreted protein pools. HT-29, SW480, DLD1 and Lovo cells were seeded in $100 \mathrm{~mm}$ plates and cultured in serum-free media for $48 \mathrm{~h}$, and then the conditioned mediums were collected and concentrated for western blotting. (B) GST-GRP78 was purified by GST affinity chromatography. (C) DLD1, SW480 and HT-29 cells were treated with $1 \mu \mathrm{M}$ either GST protein or GST-GRP78. The number of cells was determined with MTT assay. (D) DLD1 was treated with 100 nM, 200 nM, 500 nM, 1000 nM and 2000 nM GST-GRP78 for 24 hours, and the number of cells was determined with the MTT assay. The same concentration of GST protein served as controls. (E) DLD1 cells were treated with $1 \mu \mathrm{M}$ GST protein or GST-GRP78 for 2 weeks, and then cells were stained with crystal violet. (F) Quantitative analyse of colony formation efficiency. $* p<0.05, * * p<0.01$, and $* * * p<0.001$ versus the GST group.

TRIAZOL reagent. The cDNA obtained by reverse transcription was used as the template to amplify GRP78 gene. Bacterial expression plasmid was prepared by ligating the full-length GRP78 coding sequence into the Bam HI/Xho I sites of pGEX-4T-2. GRP78 fused to glutathione S-transferase (GST) were expressed in E.coli and purified by GST-affinity purification (Qi et al., 2010).

The isolated GST-GRP78 fusion protein was analysed by PAGE under non-denaturing conditions. $10 \mu \mathrm{g}$ protein was subjected to different lanes of $8 \%$ polyacrylamide gel electrophoresis without SDS at $4^{\circ} \mathrm{C}$ with a constant current of $20 \mathrm{~mA} / \mathrm{gel}$. After electrophoresis, the gel was stained with Coomassie Brilliant Blue R-250.

\section{Cell culture and conditioned medium collection}

The colon cancer cells of DLD1, SW480, HT-29 and Lovo were cultured in RPMI-1640 medium containing $10 \% \mathrm{FBS}$ at $37^{\circ} \mathrm{C}$ in humidified tissue culture incubator containing $5 \% \mathrm{CO}_{2}$. Conditioned medium from colon cancer cells was generated as follows: $80 \%$ of confluent cells were fed with serum-free DMEM and incubated for 24 hours. The medium was then collected, clarified by centrifugation for 30 minutes at $5,000 \mathrm{rpm}$ and concentrated by the $30-\mathrm{kDa}$ MW cut-off ultrafiltration membranes (Millipore).

\section{MTT and colony formation assays}

For cell growth assay, 2000 cells were seeded into a 96-well plate and treated with purified GRP78. After 24 hours treatment, $20 \mu 1 \mathrm{MTT}(5 \mathrm{mg} / \mathrm{ml})$ was added to each well to reveal cell proliferation. Colony formation assay was performed as previously reported (Yue et al., 2012). Briefly, DLD1 cells were plated onto $60 \mathrm{~mm}$ dishes at 5000 cells per dish and then treated with GST or GSTGRP78 respectively for 2 weeks at $37^{\circ} \mathrm{C}$. When large colonies were visible, the cells were fixed with methanol and stained with crystal violet for $20 \mathrm{~min}$. The numbers of colony was counted under phase-contrast microscope.

\section{Western blotting and GST pull down assay}

The whole cell extracts, as well as the concentrated cell culture supernatants were resolved by SDS-PAGE, transferred to a PVDF membrane. After incubation with primary antibodies, blots were incubated with HRPconjugated secondary antibody for 2 hours at room temperature. The bands were visualized using an enhanced chemiluminescence detection kit and radiographic film.

For GST-pull down assay, $2 \times 10^{7}$ DLD1 cells were collected and all of the proteins from cell surface were extracted according to the protocol. $300 \mu \mathrm{g}$ cell suface proteins extraction were incubated with GST and GSTGRP78 bound to glutathione-Sepharose beads for 2 hours at $4^{\circ} \mathrm{C}$ by gently shaking. The bound proteins were detected by SDS-PAGE silver nitrate stained analysis.

\section{Silver nitrate staining and mass spectrometry}

After electrophoresis, the gel slab was fixed in 50\% methanol, $5 \%$ acetic acid in water for $20 \mathrm{~min}$. It was then washed for 10 min with $50 \%$ methanol in water and another 10 min with water. The gel was sensitized by a 1 min incubation in $0.02 \%$ sodium thiosulfate. It was then rinsed with two changes of distilled water for $1 \mathrm{~min}$ each. 


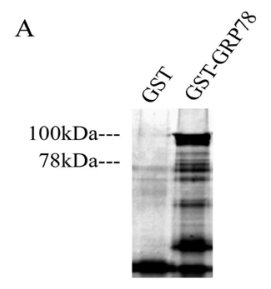

C

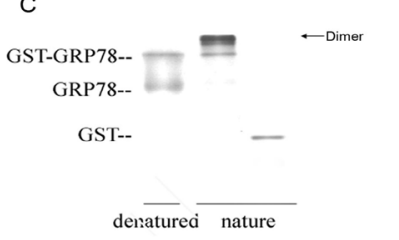

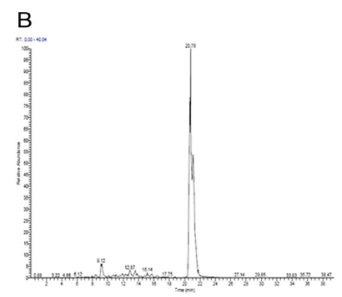

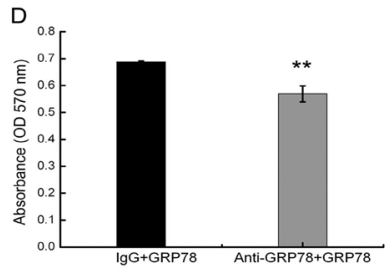

Figure 2. Secreted GRP78 is a Novel Ligand of Cell Surface GRP78 (A) GST-GRP78 pulldown of membrane lysates from DLD1 cells. The precipitates were separated by SDS-PAGE. The gel was stained by AgNO3 and interesting bands were cut for mass spectrometry. (B) Secondary mass spectrum of the gel cut from (A), X-axis stated Retention time; Y-axis stated Relative abundance. (C) PAGE under non-denaturing conditions of denatured GST-GRP78, nature GST-GRP78 and GST protein. (D) DLD1 cells were treated with anti-GRP78 antibodies or IgG for 2 hours, and then $1 \mu \mathrm{M}$ GST-GRP78 was added. Cell proliferation was examined by the MTT assay. ${ }^{*} p<0.01$ versus the $\mathrm{IgG}+\mathrm{GRP} 78$ group.

After rinsing, the gel was submerged in chilled $0.1 \%$ silver nitrate solution and incubated for $20 \mathrm{~min}$ at $4{ }^{\circ} \mathrm{C}$. After incubation, the silver nitrate was discarded. The gel slab was rinsed twice with water for $5 \mathrm{~s}$, and then developed in $6 \% \mathrm{Na} 2 \mathrm{CO} 3,0.05 \%$ formalin in $0.0004 \% \mathrm{Na} 2 \mathrm{~S} 2 \mathrm{O} 3$ with intensive shaking. Silver-stained gels were stored in a solution of $1 \%$ acetic acid at $4^{\circ} \mathrm{C}$. The spots of interest were excised from gels and subjected to mass spectrometry (Rabilloud et al., 1988; Shevchenko et al., 1996).

\section{Statistical analysis}

Data are expressed as the mean \pm SEM. Differences among groups were tested by one-way analysis of variance (ANOVA). Comparisons between two groups were evaluated using Student's t-test. A value of $p<0.05$ was considered statistically significant.

\section{Results}

Secreted GRP78 from colon cancer cells promotes cell proliferation

To investigate whether GRP78 could be secreted from colon cancer cells, the conditioned medium from HT-29, SW480, DLD1 and Lovo cells were collected and concentrated. As shown in Figure 1A, GRP78 was secreted by all the four types of colon cancer cells. Among them, DLD1 cells had the highest secretory capacity. Next, to assess the autocrine role of GRP78, the full length GRP78 gene was cloned, expressed and purified in E. coli (Figure 1B). When applied to cells in culture, GRP78 significantly promoted cell proliferation, especially in DLD1 cells, as demonstrated by the MTT assay (Figure 1C). The optimal concentration of GRP78 to promote DLD1 cell

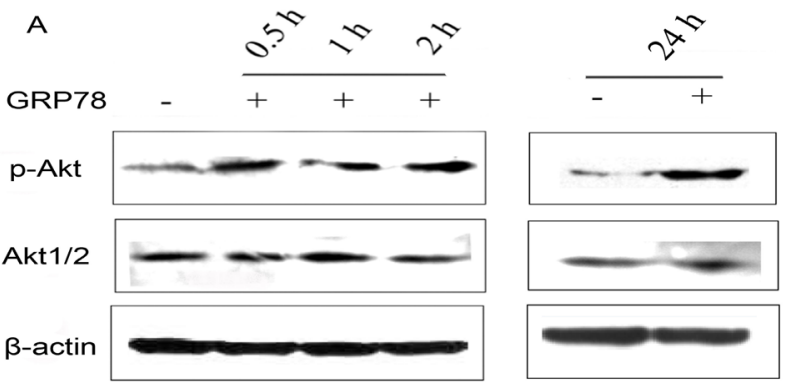

B

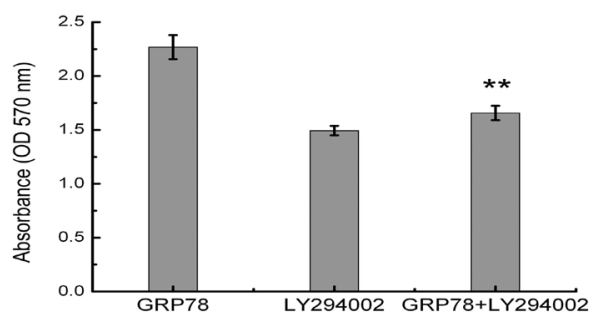

Figure 3. Secreted GRP78 Activates the PI3K/Akt Signaling Pathway (A) Western blotting analysis of $p$-Akt in DLD1 cells treated with GST-GRP78 for the indicated time intervals. (B) DLD1 cells were kept in regular growth media supplemented with $30 \mu \mathrm{M} \mathrm{LY} 294002$ for $1 \mathrm{~h}$, and then treated with GST-GRP78 for $24 \mathrm{~h}$. The number of cells was determined with the MTT assay. ${ }^{*} p<0.01$ versus GRP78.

Table 1. Mass Spectrometry Analysis of GRP78Binding Proteins

\begin{tabular}{lcccl}
\hline $\begin{array}{l}\text { Pep } \\
\text { Count } \\
\text { Sequence }\end{array}$ & $\begin{array}{c}\text { Unique } \\
\text { Pep } \\
\text { Count } \\
\text { MH+ }\end{array}$ & $\begin{array}{c}\text { MW } \\
\text { Charge }\end{array}$ & PI Rank & $\begin{array}{l}\text { Identified } \\
\text { Name XC }\end{array}$ \\
\hline 380 & 48 & 72332.86 & 5.07 & $\begin{array}{l}\text { IPI:IPI00003362.3 } \\
\text { HSPA5 }\end{array}$ \\
5 & 3 & 71704.1 & 6.33 & $\begin{array}{l}\text { IPI:IPI00022434.4 } \\
\text { ALB Uncharacterized protein } \\
\text { IPI:IPI00339269.1 }\end{array}$ \\
20 & 2 & 71028.19 & 5.81 & $\begin{array}{l}\text { HSPA6 } \\
\text { IPI:IPI00027547.2 } \\
\text { Dermcidin }\end{array}$ \\
\hline
\end{tabular}

proliferation was at $1 \mu \mathrm{M}$ (Figure 1D). Furthermore, an obvious proliferation-promoting effect was also observed using plate colony formation assay (Figure $1 \mathrm{E}$ and $1 \mathrm{~F}$ ).

Secreted GRP78 is a novel ligand of cell surface GRP78

To identify the potential signal receptor of secreted GRP78, cell membrane protein of DLD1 cell was extracted and incubated with either GST-GRP78 or GST protein, followed by GST pull down assay. The GRP78-binding proteins were then analyzed by silver staining SDSPAGE and cut for mass spectrometry (Figure 2A). The results indicated that the most probable binding protein of secreted GRP78 was the membrane GRP78 (Figure 2B and Table 1). Furthermore, GST-GRP78 protein was likely to form a dimer itself when loaded onto a nondenaturing PAGE gel (Figure 2C). Moreover, blockage of cell surface GRP78 by anti-GRP78 antibody reversed the pro-proliferative effects of GST-GRP78 (Figure 2D). The above data indicate that secreted GRP78 is able to utilize cell surface GRP78 as its signaling receptor. 


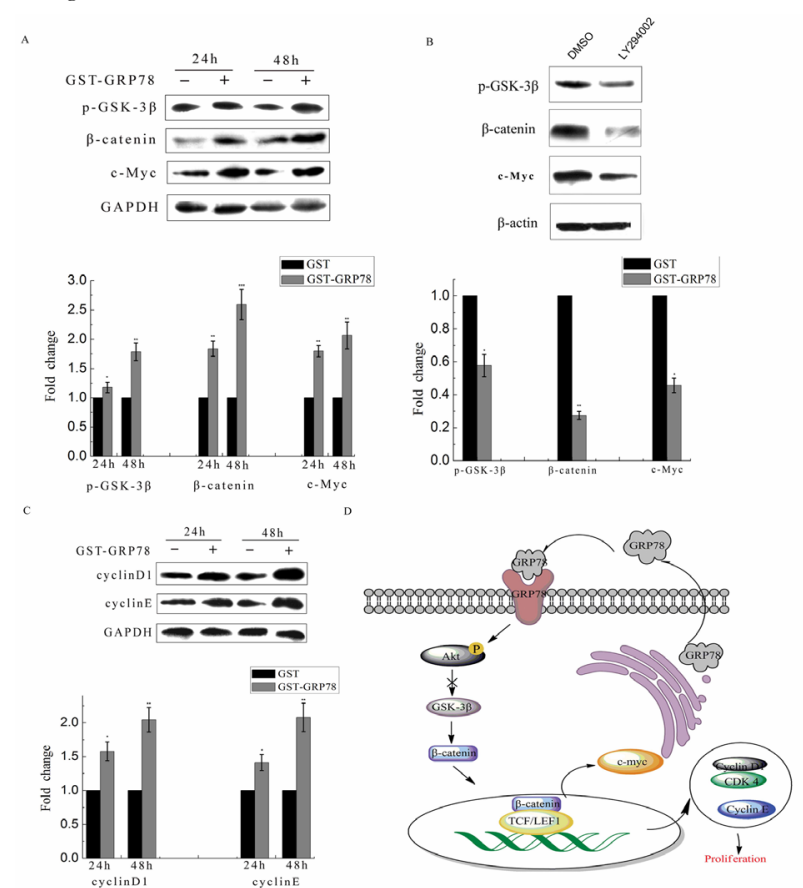

Figure 4. Secreted GRP78 Stimulates the $\beta$-catenin Signaling Pathway. (A) Western blotting analysis of $p$-GSK$3 \beta, \beta$-catenin and c-Myc in DLD1 cells treated with $1 \mu \mathrm{M}$ GSTGRP78 for $24 \mathrm{~h}, 48 \mathrm{~h}$, respectively. (B) Western blotting analysis of $p$-GSK-3 $\beta, \beta$-catenin and c-Myc in DLD1 cells treated with GST-GRP78 in the presence and absence of LY294002. (C) Western blotting analysis of cyclin D1 and cyclin E in DLD1 cells after GST-GRP78 treatment for $24 \mathrm{~h}, 48 \mathrm{~h}$, respectively. (D) Schematic representation of the proposed model. Upon microenvironmental stresses, GRP78 is overexpressed and secreted into the microenvironment. The binding of secreted GRP78 to cell surface GRP78 stimulates the PI3K/Akt signaling, which concurrently activates the $\beta$-catenin signaling and promotes cell growth.

Secreted GRP78 activates the PI3K/Akt signaling pathway

PI3K/Akt pathway is an important downstream signal of surface GRP78, mediating cell proliferation and survival (Kelber et al., 2009). GST-GRP78 treatment also elevated the phosphorylation level of Akt (Figure 3A), while treatment with $30 \mu \mathrm{M}$ LY294002, a PI3K inhibitor, significantly reversed the proliferative effect of GSTGRP78 (Figure 3B), suggesting that PI3K/Akt pathway is a downstream signal of secreted GRP78.

Secreted GRP78 promotes the activation of Wnt signaling

Aberrant activation of $\mathrm{Wnt} / \beta$-catenin signaling is implicated in malignant transformation of many cell types (Valenta et al., 2012). Activation of Akt can inactivate GSK-3 $\beta$, thus promoting the stabilization of $\beta$-catenin (Mannoury la Cour et al., 2011). Our results indicated that GSK-3 $\beta$ was phosphorylated and inactivated by GRP78 treatment. The levels of $\beta$-catenin and its downstream target gene c-Myc were also elevated by GRP78 treatment (Figure 4A). Inhibition of PI3K/Akt pathway by LY294002 reversed the above effects of GRP78, indicating that activation of $\beta$-catenin by GRP78 depends on the PI3K/Akt pathway (Figure 4B). In addition, the G1 phase-related proteins, such as cyclin D1 and cyclin E, were also raised by GRP78 treatment, suggesting that
GRP78 is likely to be implicated in regulating G1 to S progression (Figure 4C).

\section{Discussion}

Although the mortality rate declines in the past few years, colorectal cancer (CRC) remains the third most common malignancy and a major health problem worldwide (Zhu et al., 2013). Though many risk factors have been reported to be related to the CRC occurrence, the detailed pathogenesis mechanism of CRC remains not fully understood. Therefore, it is urgent to explore the mechanisms underlying CRC progression for a better prevention and therapy (Xiang and $\mathrm{Li}, 2014$ ). Besides as a molecular chaperone in ER, GRP78 is often overexpressed in tumor cells and plays an important role in tumor cell proliferation, angiogenesis, metastasis and resistance to apoptosis. Importantly, GRP78 is also considered as a prognostic biomarker in colorectal cancer (Thornton et al., 2013).

Recent evidence has shown that GRP78 can be secreted by a variety of bortezomib-resistant solid tumor cell lines, and blocks the antiangiogenic activity of bortezomib through activation of ERK $1 / 2$ but inhibition of p53 (Kern et al., 2009). Whether GRP78 can be secreted from CRC cells? If so, what are the biological functions? In the present study, we demonstrate for the first time that colon cancer cell-secreted GRP78 promotes cell proliferation in an autocrine manner. Moreover, we identify the cell surface GRP78 as the signaling receptor for secreted GRP78.

GRP78 is usually expressed on tumor cell surface and functions as a receptor of different functions, depending on its binding ligands (Gonzalez-Gronow et al., 2009). For example, ligation of surface GRP78 by $\alpha 2-\mathrm{M}^{*}$ activates MAPK and Akt-dependent signaling and promotes cellular proliferation (Misra et al., 2006), while ligation of surface GRP78 with antibodies against the C-terminal domain of GRP78 result in suppression of cell growth and induction of apoptosis (Misra et al., 2009; Misra and Pizzo, 2010a; Misra and Pizzo, 2010b). Studies have actually shown that the PI3K/Akt signaling plays a significant role in the promotion of tumor cell proliferation and colon tumorigenesis (Zheng et al., 2012). Activation of Akt can stabilize $\beta$-catenin by inactivation of GSK-3 $\beta$ or directly induce $\beta$-catenin phosphorylation, implicated in cell cycle regulation (Pandurangan, 2013). Our study shows that ligation of surface GRP78 with secreted GRP78 also activates PI3K/Akt signaling pathway and subsequently stimulates Wnt/ $\beta$-catenin pathway. Although the underlying mechanisms by which secreted GRP78 activates the PI3K/Akt signaling pathway are still unclear, it can be deduced that secreted GRP78 and $\alpha 2-\mathrm{M}^{*}$ may bind to the same domain of cell surface GRP78 in order to exert their influence.

In summary, our study identifies a novel action mode of GRP78 in tumor microenvironment: tumor cellsecreted GRP78 binds to cell surface GRP78 and activates downstream PI3K/Akt signaling pathway, consequently promoting cell proliferation and survival (Figure 4D). Our study suggests that GRP78 secretion may a measure 
taken by the tumor cells to resistant the microenvironment stresses.

\section{Acknowledgements}

This study was supported by the National Natural Science Foundation of China (No.31271516, No.31201072), Shanxi Province Science Foundation for Youths (2012021028-4). The authors declare no conflict of interest.

\section{References}

Gonzalez-Gronow M, Selim MA, Papalas J, et al (2009). GRP78: a multifunctional receptor on the cell surface. Antioxid Redox Signal, 11, 2299-306.

Kelber JA, Panopoulos AD, Shani G, et al (2009). Blockade of Cripto binding to cell surface GRP78 inhibits oncogenic Cripto signaling via MAPK/PI3K and Smad2/3 pathways. Oncogene, 28, 2324-36.

Kern J, Untergasser G, Zenzmaier C, et al (2009). GRP-78 secreted by tumor cells blocks the antiangiogenic activity of bortezomib. Blood, 114, 3960-7.

Lee AS (2007). GRP78 induction in cancer: therapeutic and prognostic implications. Cancer Res, 67, 3496-9.

Li Z, Li Z (2012). Glucose regulated protein 78: a critical link between tumor microenvironment and cancer hallmarks. Biochim Biophys Acta, 1826, 13-22.

Li Z, Zhang L, Zhao Y, et al (2013). Cell-surface GRP78 facilitates colorectal cancer cell migration and invasion. Int J Biochem Cell Biol, 45, 987-94.

Mannoury la Cour C, Salles MJ, Pasteau V, et al (2011). Signaling pathways leading to phosphorylation of Akt and GSK-3beta by activation of cloned human and rat cerebral D (2)and D (3) receptors. Mol Pharmacol, 79, 91-105.

Misra UK, Deedwania R, Pizzo SV (2006). Activation and cross-talk between Akt, NF-kappaB, and unfolded protein response signaling in 1-LN prostate cancer cells consequent to ligation of cell surface-associated GRP78. J Biol Chem, 281, 13694-707.

Misra UK, Mowery Y, Kaczowka S, et al (2009). Ligation of cancer cell surface GRP78 with antibodies directed against its $\mathrm{COOH}$-terminal domain up-regulates p53 activity and promotes apoptosis. Mol Cancer Ther, 8, 1350-62.

Misra UK, Pizzo SV (2010a). Ligation of cell surface GRP78 with antibody directed against the $\mathrm{COOH}$-terminal domain of GRP78 suppresses Ras/MAPK and PI 3-kinase/AKT signaling while promoting caspase activation in human prostate cancer cells. Cancer Biol Ther, 9, 142-52.

Misra UK, Pizzo SV (2010b). Modulation of the unfolded protein response in prostate cancer cells by antibody-directed against the carboxyl-terminal domain of GRP78. Apoptosis, 15, 173-82.

Pandurangan AK (2013). Potential targets for prevention of colorectal cancer: a focus on PI3K/Akt/mTOR and Wnt pathways. Asian Pac J Cancer Prev, 14, 2201-5.

Qi RF, Liu ZX, Xu SQ, et al (2010). Small peptides derived from the Lys active fragment of the mung bean trypsin inhibitor are fully active against trypsin. FEBS J, 277, 224-32.

Rabilloud T, Carpentier G, Tarroux P (1988). Improvement and simplification of low-background silver staining of proteins by using sodium dithionite. Electrophoresis, 9, 288-91.

Shani G, Fischer WH, Justice NJ, et al (2008). GRP78 and Cripto form a complex at the cell surface and collaborate to inhibit transforming growth factor beta signaling and enhance cell growth. Mol Cell Biol, 28, 666-77.

ShevchenkoA, Wilm M, Vorm O, et al (1996). Mass spectrometric sequencing of proteins silver-stained polyacrylamide gels. Anal Chem, 68, 850-8.

Thornton M, Aslam MA, Tweedle EM, et al (2013). The unfolded protein response regulator GRP78 is a novel predictive biomarker in colorectal cancer. Int J Cancer, 133, 1408-18.

Valenta T, Hausmann G, Basler K (2012). The many faces and functions of beta-catenin. EMBO J, 31, 2714-36.

Vlashi E, Lagadec C, Vergnes L, et al (2011). Metabolic state of glioma stem cells and nontumorigenic cells. Proc Natl Acad Sci USA, 108, 16062-7.

Wang CM, Li SJ, Wu CH, et al (2014). Transient knock down of Grp78 reveals roles in serum ferritin mediated proinflammatory cytokine secretion in rat primary activated hepatic stellate cells. Asian Pac J Cancer Prev, 15, 605-10.

Xiang KM, Li XR (2014). MiR-133b acts as a tumor suppressor and negatively regulates TBP11 in colorectal cancer cells. Asian Pac J Cancer Prev, 15, 3767-72.

Yue P, Zhang X, Paladino D, et al (2012). Hyperactive EGF receptor, Jaks and Stat 3 signaling promote enhanced colonyforming ability, motility and migration of cisplatin-resistant ovarian cancer cells. Oncogene, 31, 2309-22.

Zheng J, Hu JD, Chen YY, et al (2012). Baicalin induces apoptosis in leukemia HL-60/ADR cells via possible downregulation of the PI3K/Akt signaling pathway. Asian Pac J Cancer Prev, 13, 1119-24.

Zhu Q-C, Gao R-Y, Wu W, et al (2013). Epithelial-mesenchymal transition and its role in the pathogenesis of colorectal cancer. Asian Pac J Cancer Prev, 14, 2689-98. 